\title{
Pericarditis y Vasculitis Secundarias a la Administración de Propiltiouracilo
}

\author{
Dra. Andrea Gleisner E.; Dra. Vera Withelm P.1 \\ Pericarditis, Vasculitis and Granulocytopenia Associated \\ to Propylthiouracil in Thyrotoxic Goiter
}

\begin{abstract}
A case of adverse reaction to propylthiouracil therapy in a child with thyrotoxic nodular goiter is reported. After 26 months of well tolerated therapy he had fever, joint pain, swelling of legs and evidence of pericarditis. Subsequently there appeared high grade fever, malaise, purpuric skin lesions, conjunctivitis, granulocytopenia, adenopathies and splenomenaly There were not clinical nor laboratory siens of associated intercurrent diseases. Complete remission of symptoms on withdrawal of propyl thiouracil aLlowed us to make a diagnosis of an adverse reaction to the drug with pericarditis, vasculitis and granulocytopenia.

(Key words: Propylthiouracil Untoward reactions. Pericarditis, Granulocytopenia. Vasculitis).
\end{abstract}

El bocio multinodular se observa en general en el sexo femenino en la quinta década de la vida. En el adulto se asocia frecuentemente con hiper. tiroidismo, lo que en el niño y en el adolescente es excepcional. Cuando esto ocurre en la infancia, los signos clínicos de hipertiroidismo son extremadamente discretos y a veces sólo las mediciones hormonales permiten hacer el diagnóstico. El tratamiento médico preconizado en el bocio simple puede ser aplicado en el bocio multinodular. En ausencia de remisión y si hay signos de compresión cervical está indicada la extirpación quirúrgica.

En los casos de bocio multinodular hipertiroideo el tratamiento inicial se hace mediante antitiroídeos de sintesis $y$, eventualmente, extirpación quirúrgica.

Se presenta un caso de bocio multinodular acompañado de hipertiroidismo, tratado inicialmente con propiltiouracilo (PTU) que debió suspenderse por reacción alérgica al medicamento.

\section{Caso Clínico}

Varón, consultó a los 9 años de edad por aumento de volumen en región anterior del cuello de dos meses de evolución. Desarrollo pon-

1. Departamento de Pediatría, Facultad de Medicina, Univer sidad de Concepción. doestatural normal. Sin afecciones previas de importancia. En el examen físico se encontró un bocio multinodular de predominio derecho, sin signos clínicos de hipertiroidismo.

La captación de 1131 fue, en 24 horas, $17 \%$ y se mantuvo sin variación luego de la prueba de supresión. Cintigrama de tiroides: captación djfusa; algo irregular y un nódulo caliente en el. lóbulo derecho, cuya imagen no se modificó después de la prueba de supresión. Tiroxina plasmática (T4): $16,5 \mu \mathrm{g} \mathrm{x}$ dl. Triiodotiroxina plasmática (T3): $445 \mathrm{ng} x$ dl. Fue tratado con Propiltiouracílo (PTU) $200 \mathrm{mg} x$ día por 3 meses, luego se redujo la dosis a $150 \mathrm{mg} x$ día, con buena tolerancia clínica y sin modificación del bocio.

Cuando habían transcurrido 26 meses de tratamiento presentó edema y artralgia de las manos, que cedieron espontáneamente. Cuatro meses después tuvo artralgia de rodillas, decaimiento, fiebre, baja de peso, eritema nodoso, polipnea, taquicardia y frotes pericárdicos, sin compromiso valvular (Figura 1). Se suspendió PTU. La radiografía de tórax, el electrocardiograma y el ecocardiograma confirmaron que había derrame pericárdico. Mediante pericardiocentesis se evacuaron $400 \mathrm{cc}$ de líquido hemorrágico, cuyos cultivos bacteriológicos y estudios para bacilo de Koch fueton negativos. Esta vez las mediciones de hormonas tiroídeas mostraron $\mathrm{T} 4$ : 16,4 $\mu \mathrm{g} x \mathrm{dl}$, T3: $450 \mathrm{ng} \mathrm{x}$ dl y Tiroestimulina hipofisiaria (TSH): $<1 \mu \mathrm{IU} / \mathrm{ml}$. Anticuerpos antitiroglobulina $y$ antimicrosomales negativos. Velocidad de sedimentación globular (VHS) 100 


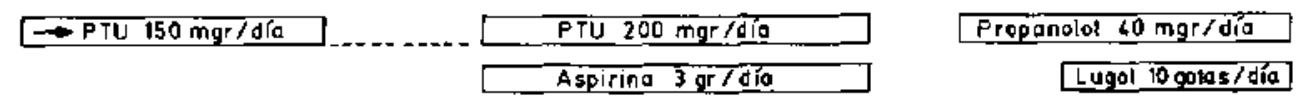

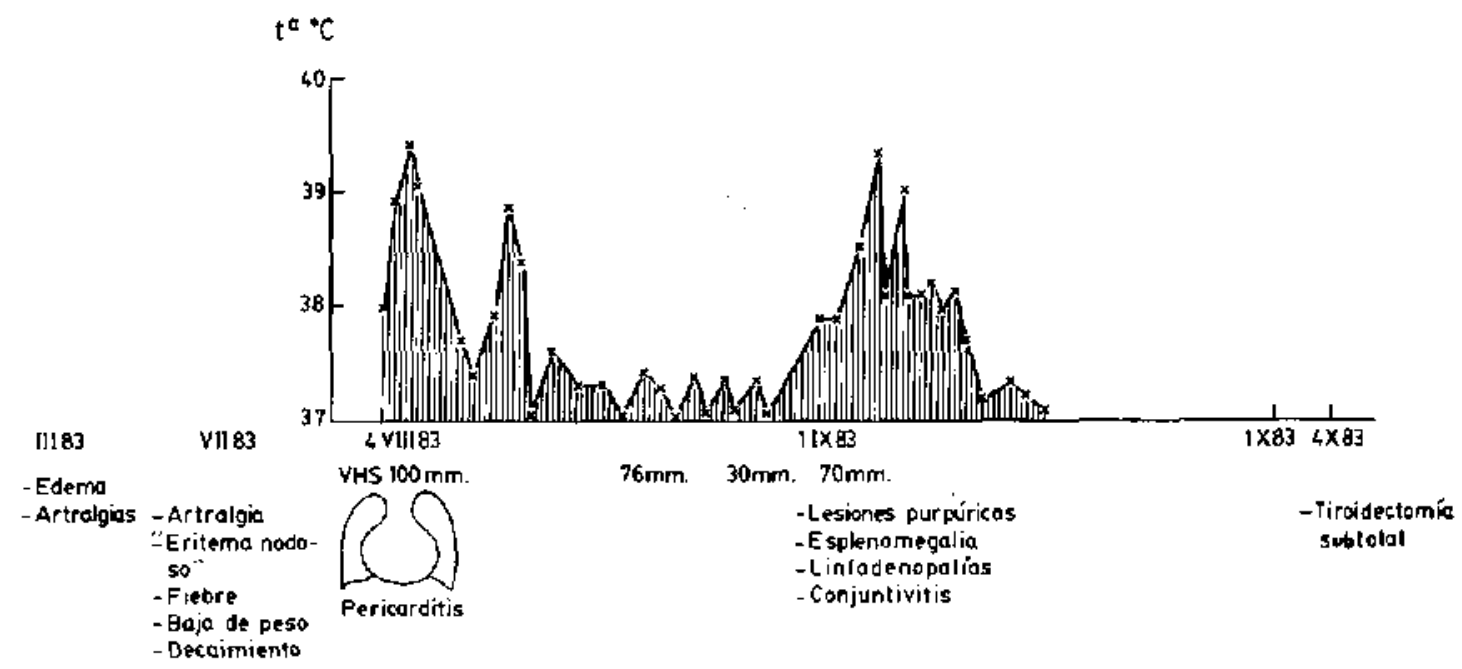

Figura 1. Evolución clínica de un paciente varon de 11 años de edad con reacción adversa al PTU.

mm en la lra. hora. Hemogtama normal. Título antiestreptolisina ' $\mathrm{O}^{\prime}$ ( $\left.\mathrm{ASO}\right)>800 \mathrm{U}$.

Pensando que sufría de enfermedad reumática se indico reposo, erradicación estreptocócica, aspirina y se reinició el tratamiento con PTU.

La evolución clínica fue satisfactoria, desapareciendo los síntomas en una semana. Sin embargo, tres semanas después, nuevamente presentó fiebre alta, conjuntivitis, adenopatías múlti. ples, esplenomegalia y lesiones cutáneas máculo. papulares purpúricas, dolorosas, localizadas en pabellón auricular, región malar y antebrazo (Figura 2). La VHS aumentó nuevamente y en el hemograma apareció granulocitopenia. Los anticuerpos antinucleares y el factor reumatuideo dieron resultados negativos. Habiendo descartado un proceso séptico, se suspendió PTU en coincidencia con lo cual desaparecieron todos los signos y síntomas descritos. Posteriormente se realizó tiroidectomía subtotal bajo terapia con lugol y propanolol. El estudio histológico de la pieza extirpada confirmó un bocio multinodular hipertiroídeo. Finalmente se indicó tratamiento de sustitución, con Levotiroxina.

\section{DISCUSION}

Los antitiroídeos de sintesis (PTU, metimazole) son los más utilizados en los niños por su

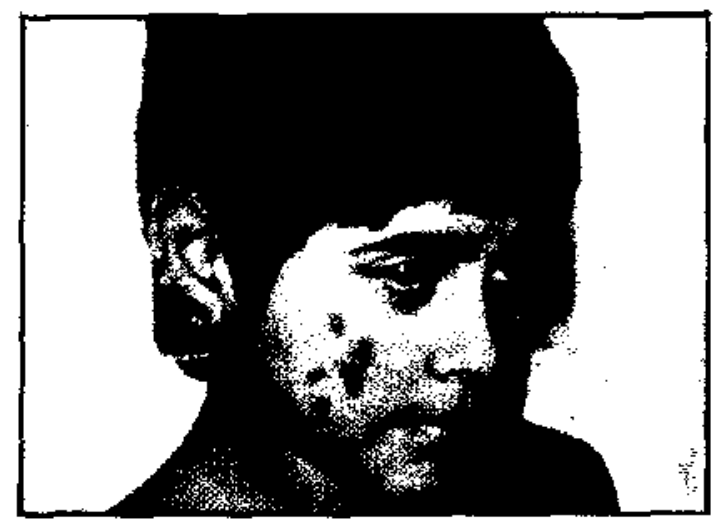

Figura 2. Lesiones cutáneas máculopapulares purpúricas en pabellón auricular $y$ región malar secundarias a la administración de PTU.

eficacia y fácil administración, siendo excepcional que produzcan complicaciones graves ${ }^{1,} \hat{2}, \mathbf{3}$, 4. Sin embargo se han descrito reacciones medicamentosas en la mayoría de las grandes series de pacientes tratados con antitiroideos 5,6 ,

Las complicaciones secundarias a la adminis. tración de tioamidas aparecen aproximadamente en 3 a $7 \%$ de los casos y cesan luego de suspender la terapia. Estas incluyen granulocitopenia, trombocitopenia, eritemas múltiples, urticarias, hepatitis, fiebre y prurito generalizado ${ }^{1,3,4}$, En 
otros casos se presentan con características semejantes al síndrome lúpico ${ }^{7}, \mathbf{a}, 10$. Los efictos colaterales se observan generalmente en las primeras semanas de tratamiento 1,10 .

Existen reacciones cruzadas entre las diversas drogas. Si no se reconocen las reacciones adversas a las drogas antititoides y continúa la exposición del paciente a ellas, los resultados pueden ser potencialmente fatales ${ }^{11}, 12.13$.

E1 compromiso cutáneo est í representado generalmente por una erupción fugaz urticarial, que desaparece en forma espontánea. Se describen además erupciones cutáneas purpúricas, palpables, que pueden localizarse en el lóbulo del pabellón auricular, en la región malar, en las extremidades o ambas, que pueden ser dolorosas y corresponden a una vasculitis inducida por la droga $3,2,4,10,11,14$.

En nuestro paciente las artralgias migratorias, la fiebre y la pericarditis, sugerían que sufría de enfermedad reumática, posibilidad apoyada, además, por VHS y títulos de ASO elevados. No se pensó inicialmente en una reacción secundaria el PTU en razón al largo tiempo transcurrido desde el comienzo del tratamiento (26 meses).

La remisión de los síntomas coincidiendo con el reposo, el uso de aspirina y penicilina, probablemente tuvo su origen en la supresión del tratamiento con PTU, ya que, tres semanas después de reanudado este último aparecieron nuevas manifestaciones alérgicas cuya desaparición total coincidió con la supresión definitiva de la droga.

\section{RESUMEN}

Se describe un paciente de 11 años de edad con bocio multinodular hipertiroídeo que des. pués de 26 meses de tratamiento con Propiltiouracilo sufrió una reacción adversa con la droga, con evidencias de pericarditis, vasculitis y granulocitopenia. Los sintomas desaparecieron rápidamente al suspender la droga. Posteriormente fue sometido a tiroidectomia y tratamiento hormonal de reemplazo.

\section{REFERENCIAS}

1. Cooper, D.: Antithyroid drugs. N. Engl, J, Med. 311: $1353,1984$.

2. Vasily D., Tyler $w_{1}$ : Propilthiouracil - induced cutancous vasculitis. Case presentation and review of the liferature. JAMA 243: 458, 1980.

7 Goopcr D., Goldminz D., Lewin. A., Ladenson P., Daniels G., Molitch M. Ridgway Ch.: Agramulocítosis associated with antithyroid drugs. Ann. Intern. Mcd. 98: 26, 1983.

4. Cassorla F., Finegold D., Parks J., Tenore A., Thoweroni H., Baker L.: Vasculitis, pulmonary cavitation, and anemia during antithyroid drug therapy. Am. J. Dis. Childa. 137: $118,1983$.

5. Barnes, H.V., Blizzard, R.M.: Antithyroid drug therapy for toxic diffuse goiter (graves disease); Thirty years experience in children and adoles. centes. J. Pexdiatr. 91: 313, 1977.

6. Voidya, V.A., Bongiovami, A.M., Porks, J.S., Tenor, A., Kirkland. F.F.: Twenty-two years experience in the medical management of juvenile thyrotoxicosis. Pediatrics, 54:30, 1974.

7. Hung W., August G.: A. "Collagen - like" Syndrome associated with antithyroid therapy. J. Pediatr. 82: $852,1973$.

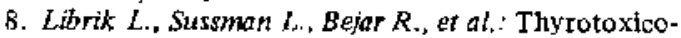
sis and collagen - like disease in three sisters of American Indian extraction, J. Pediatr. 76: 64, 1970.

9. Amrhein J., Kenny F, and Ross D.: Granulocytopenia, lupus like syndrome and other complications of Propylthiouracil therapy. Pediatrics 76: 54, 1970.

10. Sammon T., Peden V., Wirzleben C. King J.: Disseminated intravascular coagulation complicating propylthiouracil therapy. Clin. Pediatr. 10:739, 1971 .

11. Reidy $T$., Upshaw d., Chesney T.: Propylthiouracil - induced vasculitis: a fatal case. South Med. J. 75: $1297,1982$.

12. Mc Conick $R$.: Periarteritis occuring during Propylthiouracil Therapy. JAMA 144: 1453, 1950.

13. Miyazono, $K$., Okazaki, T., Uchida. S., Totsuka, $I$., Matsumoto, T.: Propylthioyracil - induced diffuse intersticial Pneumonitis. Arch. Intern. Med. 144: $1764,1184$.

14. Koustan B., Crouch M. Brick J and Di Bartobmeo A.: Apparent vasculitis associated with propylthiouracil use. Arthritis. Rheum. 22: 925, 1979. 